\title{
Assistência de enfermagem quanto ao paciente ortopédico em um hospital público do oeste do Pará
}

Nursing care for orthopedical patients in a public hospital in west Pará

Atención de enfermería a pacientes ortopédicos en un hospital público de Pará occidental

Sílvia Maria Farias dos Santos

ORCID: https://orcid.org/0000-0001-8360-6267

Universidade Estadual do Pará, Brasil

E-mail: silvia.farias.enf@gmail.com

Maria Monica Machado de Aguiar Lima

ORCID: https://orcid.org/0000-0002-9164-3225

Universidade Estadual do Pará, Brasil

E-mail: monicaaguiarstm@hotmail.com

Luana Almeida dos Santos

ORCID: https://orcid.org/0000-0002-4818-1010

Universidade Federal do Oeste do Pará, Brasil

E-mail: luanah.orix@hotmail.com

Erli Marta Reis da Silva

ORCID: https://orcid.org/0000-0001.9808-4844

Universidade Estadual do Pará, Brasil

E-mail: erlimartareis@ hotmail.com

Antenor Matos de Carvalho Junior

ORCID: https://orcid.org/0000-0003-3507-7932

Universidade Estadual do Pará, Brasil

E-mail: mattos.antenorjr@gmail.com

Rodrigo Ruan Costa De Matos

ORCID: https://orcid.org/0000-0001-7943-5282

Universidade Estadual do Pará, Brasil

E-mail: ruandmatos@gmail.com

\section{Resumo}

O Sistema de Classificação de Pacientes caracteriza a necessidade e o grau de dependência do paciente em relação aos cuidados da equipe, sendo a ferramenta que auxilia na sistematização da assistência, contribuindo para o desenvolvimento do cuidado individualizado e integral. Assim, o estudo teve como objetivo investigar a atuação do enfermeiro mediante o grau de dependência do paciente vítima de trauma ortopédico. Trata-se de um estudo descritivo, com corte transversal, e qualitativa, teve como participantes 4 enfermeiros. Os dados qualitativos foram tratados através do software IRAMUTEQ (Interface de R por Análises Multidimensionais de Texto e Questionários). $\mathrm{O}$ corpus do texto deu origem a seis classes temáticas, que foram agrupadas e intituladas de acordo com sua aproximação, subsidiando a construção de quatro categorias de análise: a visão e prática do enfermeiro frente ao sistema de classificação de pacientes, a prática do enfermeiro frente ao dimensionamento de pessoal, o reflexo do número reduzido de profissional na assistência e orientações direcionadas ao paciente na alta hospitalar.

Palavras-chave: Assistência de enfermagem; Traumas; Ortopedia.

\begin{abstract}
The Patient Classification System characterizes the patient's need and degree of dependence on the care of the team, being the tool that helps in the systematization of care, contributing to the development of individualized and comprehensive care. Thus, the study aimed to investigate the role of nurses according to the degree of dependence of the patient victim of orthopedic trauma. This is a descriptive, cross-sectional, and qualitative study, with 4 nurses participating. Qualitative data were treated using the IRAMUTEQ software (R Interface for Multidimensional Text Analysis and Questionnaires). The text corpus gave rise to six thematic classes, which were grouped and titled according to their approach, supporting the construction of four categories of analysis: the view and practice of nurses in the face of the patient classification system, the practice of nurses in front of to staff dimensioning, the reflection of the reduced number of professionals in care and guidance directed to the patient at hospital discharge.
\end{abstract}

Keywords: Nursing care; Traumas; Orthopedics. 


\section{Resumen}

El Sistema de Clasificación de Pacientes caracteriza la necesidad del paciente y el grado de dependencia de la atención del equipo, siendo la herramienta que ayuda en la sistematización de la atención, contribuyendo al desarrollo de una atención individualizada e integral. Así, el estudio tuvo como objetivo investigar el papel de las enfermeras según el grado de dependencia del paciente víctima de traumatismo ortopédico. Se trata de un estudio descriptivo, transversal y cualitativo, con la participación de 4 enfermeras. Los datos cualitativos se trataron con el software IRAMUTEQ (Interfaz R para Análisis de Texto Multidimensional y Cuestionarios). El corpus de textos dio lugar a seis clases temáticas, que fueron agrupadas y tituladas según su enfoque, apoyando la construcción de cuatro categorías de análisis: la mirada y práctica del enfermero frente al sistema de clasificación de pacientes, la práctica del enfermero frente al sistema de clasificación de pacientes. del dimensionamiento del personal, el reflejo del reducido número de profesionales en la atención y orientación dirigida al paciente al alta hospitalaria.

Palabras clave: Atención de enfermeira; Traumas; Ortopedía.

\section{Introdução}

O trauma ortopédico é uma das condições mais mórbidas existentes na sociedade contemporânea, comprometendo a função do indivíduo, sua participação econômica na sociedade. Os profissionais dedicados ao atendimento do trauma ortopédico precisam receber não apenas a formação elementar em ortopedia e traumatologia. Mas, idealmente, treinamento complementar para a tomada de decisões em situações complexas (Kfuri, 2021).

Neste sentido utilizado na prática profissional para implementar uma teoria de enfermagem. Este método destaca-se por necessitar da investigação contínua dos fatores de risco e de bem estar que envolvem o paciente, até quando não há problemas evidentes. O processo de enfermagem (PE), permite que o enfermeiro desenvolva um trabalho amparado por modelos de cuidados, fazendo-o praticar o pensamento crítico, tornando-se essencial na deliberação e tomada de decisão (Barros; Lopes, 2010).

Wanda de Aguiar Horta em 1960, baseada em sua teoria apresentou um modelo de PE com seis etapas: histórico de enfermagem, diagnóstico de enfermagem, planejamento assistencial, planos de cuidados ou prescrição de enfermagem, evolução e prognóstico de enfermagem (Horta, 1979).

"Processo de enfermagem é o paradigma científico, sem alternativa até o momento atual, de que a enfermagem deve lançar mão para ser reconhecida e consolidada como ciência". Alguns enfermeiros têm usado este processo como método para sistematizar a assistência de enfermagem nos diversos níveis assistenciais, desenvolvendo-se em cinco etapas sequenciais e relacionadas, que ocorrem concomitantemente, sendo elas: investigação, diagnóstico de enfermagem, planejamento, implementação da assistência de enfermagem e avaliação (Tannure; Pinheiro, 2013).

Desde a década de 80, a Sistema Assistência na Enfermagem (SAE) tem sido uma determinação legal no Brasil, instituída pela primeira vez na lei 7.498/86, que dispunha sobre a regulamentação do exercício profissional da enfermagem (Cofen, 1987).

A resolução COFEN n 358/2009 afirma que a implantação do SAE deve ocorrer em todas as instituições de saúde, públicas e privadas. É uma atividade privativa do enfermeiro, devendo este realizar a implementação, o planejamento, a organização, a execução e a avaliação do processo de enfermagem (Cofen, 2009).

Atualmente, a SAE vem sendo amplamente estudada e aplicada nos serviços de saúde no Brasil e no mundo. No Brasil o modelo proposto por Wanda Horta, é o mais conhecido e utilizado para a implementação do processo de enfermagem. Na prática clínica observa-se que a sistematização da assistência de enfermagem é adotada em apenas alguns serviços de saúde, sendo mais presente do discurso profissional do que na vivencia cotidiana. Porém, isso pode ser explicado pelo fato de haver alguns obstáculos que interferem na execução da sistematização da assistência como: mão de obra escassa, falta de tempo para o preenchimento da documentação escrita, demanda elevada para o número de profissional, entre outros (Barros; Lopes, 2010). 
O Sistema de Classificação de Pacientes (SCP), consiste na observação do cuidado individualizado de acordo com a necessidade do paciente, agrupando-o em categorias conforme seu grau de complexidade, esse sistema é considerado útil no gerenciamento e no planejamento da assistência, uma vez que subsidia o dimensionamento dos membros da equipe de enfermagem e a alocação de profissionais capacitados, organização de materiais adequados e instalações adaptadas, além de potencializar as ações educativas específicas, contribuindo assim para a melhoria da assistência prestada, aumentando a satisfação dos usuários e familiares (Brito; Guirardello, 2012).

Segundo Moraes, Linch e Souza (2012), visando contribuir para a melhora da qualidade da assistência prestada ao paciente vítima de trauma é importante adotar um sistema de classificação para estes pacientes, principalmente no período pré e pós-operatório, pois eles sofrem com o impacto da redução ou da perda das funções vitais, sendo altamente dependentes dos cuidados de enfermagem.

Para estimar a carga de trabalho da enfermagem, podem ser utilizadas ferramentas diretas ou indiretas para obtenção das horas de cuidado, necessárias para subsidiar o cálculo de dimensionamento de profissionais. Atualmente no Brasil, tem-se utilizado de Sistema de Classificação de Pacientes, os quais estipulam as horas de cuidados de acordo com a complexidade assistencial do paciente (Ferreira et al., 2017).

Conforme a resolução COFEN 543 de 2017, o dimensionamento do quadro de profissionais de enfermagem deve estar baseado em características relativas ao serviço de saúde, ao serviço de enfermagem e ao paciente. Depois de realizada a etapa de classificação de pacientes, para seguir na definição da carga média de trabalho da unidade, é preciso definir o quantitativo, mínimo de horas necessário para prestar assistência de enfermagem conforme cada tipo de cuidado, para efeito de cálculo, devem ser consideradas como horas de enfermagem, por leito, nas 24 horas:

Assim observa-se a relevância do dimensionamento de pessoal adequado isto se deve ao fato de que o subdimensionamento poderá acarretar sobrecarga de trabalho e consequentemente o aumento dos eventos adversos, enquanto o superdimensionamento aumenta sem necessidade o ônus hospitalar, porém, sem influenciar no aumento da qualidade da assistência ofertada (Fugulin et al, 2012).

\section{Metodologia}

Trata-se de uma pesquisa descritiva, com corte transversal, e abordagem qualitativa. Segundo Prodanov e Freitas (2013), a pesquisa descritiva tem como objetivo descrever características de uma população, fenômeno, ou a relação existente entre ambas. A coleta de dados neste tipo de pesquisa se dá através de um instrumento técnico padronizado.

Esta pesquisa foi desenvolvida no Hospital Municipal de Santarém (HMS), a clínica de ortopedia e traumatologia (COT) n qual foi desenvolvida a pesquisa, atualmente dispõe de 16 leitos, sendo estes destinados a pacientes vítimas de trauma ortopédico. Porém há momentos em que estes leitos são ocupados por pacientes de outras especialidades. Tendo no corpo de enfermagem: 4. Os dados foram coletados no período de 01/06/2019 à 30/07/2019 e foi realizada nas seguintes etapas:

Em seguida, foi realizada uma entrevista semiestruturada direcionada a cada enfermeiro do setor. Esta entrevista foi gravada tendo duração de aproximadamente 5 minutos, e posteriormente transcrita para o programa Microsoft Word para que pudesse ser analisada de acordo com o método análise de conteúdo segundo Bardin, (2011). Ainda direcionado aos enfermeiros, foi aplicado um questionário para caracterizar o profissional participante da pesquisa.

Os dados textuais derivados da entrevista realizada com o enfermeiro foram transcritas para o programa Microsoft Word e posteriormente tratados através do Software IRAMUTEC (Interface de $R$ pour les Analyses Multidimensionnelles de Textes et de Questionnaires) versão 0.7 alfha 1 que trata-se de uma ferramenta de processamento de dados. Este Software é gratuito na lógica de open source-código aberto. Permite que sejam realizados cinco tipos de análise: 
estatísticas textuais clássicas, classificação hierárquica descendente, pesquisa de especificidades de grupo, nuvem de palavras e análise e similitude.

A presente pesquisa foi direcionada de acordo com os princípios da Resolução 466/12 do Conselho Nacional de Saúde que contêm diretrizes e normas regulamentadoras de pesquisas envolvendo seres humanos, sendo submetido ao Comitê de Ética e Pesquisa da Universidade do Estado do Pará (UEPA) localizado na Avenida Plácido de Castro, nº 1399, bairro Aparecida, Santarém - Pará, e somente após liberação do parecer de aprovação foi dado início a coleta de dados. CAAE: 8497519.8.0000.5168, Número do Parecer: 3.263.765.

\section{Resultados e Discussão}

Essa pesquisa teve como composição de seu corpus geral 4 textos, separados em 56 segmentos de textos (ST), tendo o aproveitamento de 46 STS (80.36\%). Surgiram 1164 palavras, vocábulos ou formas, sendo destas 420 palavras distintas e 247 palavras que emergiram uma única vez. O Software categorizou o corpus analisado através da Classificação Hierárquica Descendente em 6 classes, apresentadas conforme o gráfico denominado Dendograma (Figura 1).

Figura 1- Dendograma da Classificação Hierárquica Descendente.

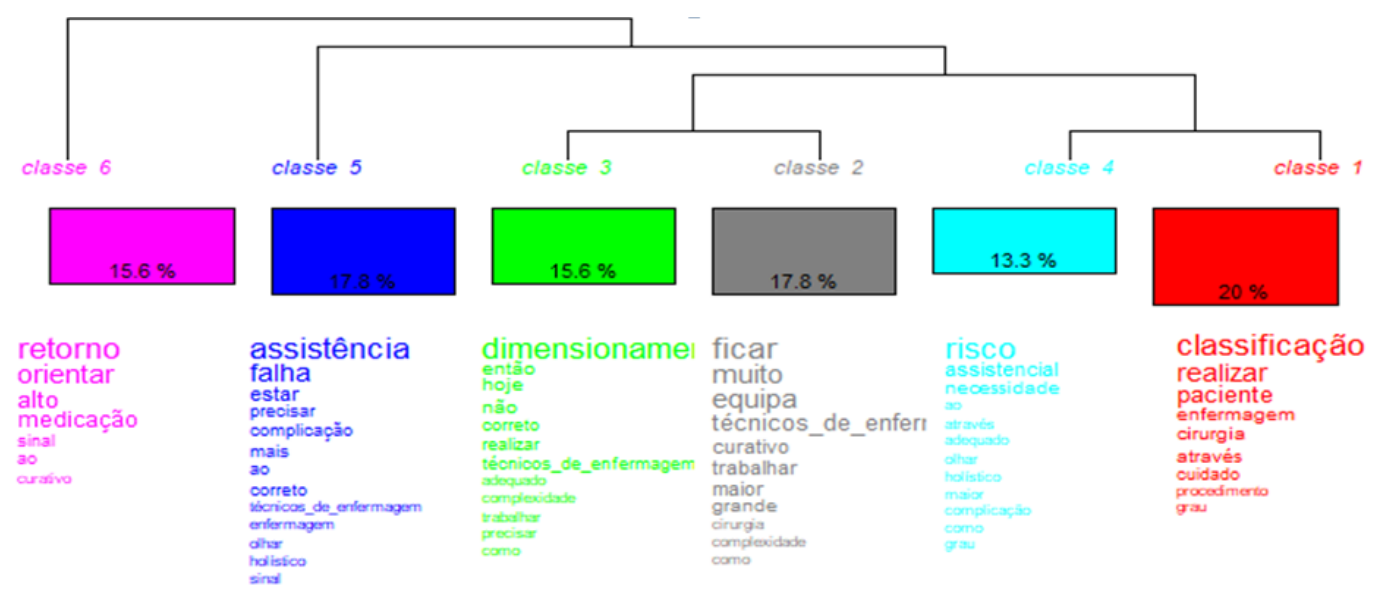

Fonte: Autores (2019).

Ressalta-se que o software dividiu o corpus em 3 eixos temáticos que deram origem a seis classes temáticas, que foram agrupadas e intituladas de acordo com sua aproximação, subsidiando a construção de quatro categorias de análise que iremos discutir a seguir.

Classe 1 e 4: A visão e prática do enfermeiro frente ao sistema de classificação de pacientes.

Classe 2 e 3: A prática do enfermeiro frente ao dimensionamento de pessoal.

Classe 5: O reflexo do número reduzido de profissional na assistência.

Classe 6: Orientações direcionadas ao paciente na alta hospitalar.

\section{Classe 1 e 4: A visão e prática do enfermeiro frente ao sistema de classificação de pacientes.}

$\mathrm{Na}$ classe 1 as palavras mais recorrentes foram "classificação" "realizar" "paciente" "enfermagem" "cirurgia" "através" "cuidado" "procedimento" "grau". Na classe 4 tem-se as palavras "risco" "assistencial" "necessidade" "através" "adequado" "olhar" "holístico" "maior" "complicação" "grau”. As sentenças do discurso retratam uma categoria onde se 
observa que o cotidiano do enfermeiro é envolto à preocupação com o cuidado e a assistência dispensada ao paciente e para isso faz uso de ferramentas que assegurem e melhorem a qualidade do cuidado. Como podemos observar nos diálogos abaixo:

(...) "eu realizo a capacitação da equipe para que o cuidado seja realizado corretamente e não traga danos para o paciente” (E1).

(...) "o sistema de classificação realizado pelo profissional de enfermagem é muito importante, através dele posso avaliar a necessidade de cuidado assistencial do paciente” (E4).

(...) "as cirurgias mais complexas, procuro realizar todos os procedimentos que serão necessários para o cuidado do paciente” (E1).

Pode-se observar que o enfermeiro da COT, entende a necessidade da realização do Sistema de Classificação do Paciente e sua importância frente ao cuidado. Segundo Gaidzinski (1994), o SCP é uma maneira de definir o grau de dependência do paciente em relação a enfermagem, e a partir da sua realização é possível calcular o tempo de cuidado direto e indireto dispensado ao paciente.

Pode-se observar na fala do E1 que o enfermeiro utiliza o tipo de cirurgia realizada pelo paciente para classificálo e assim melhor dispensar o cuidado. Vigna e Perroca (2007) observaram em seu estudo que o profissional enfermeiro utilizava outras formas de avaliação e classificação do paciente, como o acompanhamento diário da enfermagem e as informações sobre a patologia e diagnóstico médico.

Porém, apesar do enfermeiro entender a necessidade do SCP, há empecilhos que acabam dificultando sua execução durante a prática assistencial. O que pode ser observado através das falas abaixo:

(...) "devido à demanda de trabalho na clínica ser excessiva acabo não conseguindo realizar a classificação do grau de complexidade do paciente” (E4).

(...) “não realizo a classificação da complexidade do paciente principalmente pela demanda ser muito grande e eu fico responsável por dois setores, às vezes até em quatro setores, como ontem eu fiquei” (E3).

Nas falas supracitadas pode-se observar que para o profissional enfermeiro a demanda de trabalho da clínica é aumentada de tal maneira que isso o impede de realizar o sistema de classificação do paciente, porém, o enfermeiro precisa utiliza-se justamente dessa ferramenta para realizar o cálculo de dimensionamento de pessoal e assim poder encontrar o número exato de profissional enfermeiro e técnico necessário para atender a demanda da UI (Moraes; Linch; Souza, 2012).

Foi possível observar ainda através dos relatos a preocupação do enfermeiro com o processo assistencial de maneira global e em alguns momentos quando questionado quanto à maneira que utiliza para classificar o paciente em relação ao grau de complexidade, o mesmo transparece em sua fala a ligação da classificação do risco assistencial com o grau de complexidade assistencial do paciente. O que pode ser verificado no discurso abaixo:

(...) "então o paciente é classificado quanto ao risco de lesão por pressão, risco de queda e risco de broncoaspiração, também conhecidos como riscos assistenciais" (E2).

(...) "posso verificar a quais riscos assistenciais ele tá exposto e tentar evitá-los, através dele posso ainda tá realizando a sistematização da assistência, mas não consigo realizar devido a sobrecarga de trabalho” (E4).

Apesar de o sistema de classificação de pacientes fornecer de maneira indireta dados que possam facilitar e auxiliar na classificação dos riscos assistências, é preciso entender que trata-se de dois métodos diferentes de avaliação do paciente. Sendo o SCP um método através do qual vai se estabelecer a necessidade, ou a dependência do paciente em relação à 
equipe de enfermagem. Já a classificação do risco assistencial visa identificar os riscos aos quais o paciente está exposto para que a partir disso, se possa traçar um plano de cuidado que evite ou diminua o máximo possível o rico assistencial identificado durante a visita do enfermeiro.

\section{Classe 2 e 3: A prática do enfermeiro frente ao dimensionamento de pessoal}

Neste contexto as palavras mais presentes foram "ficar" "muito" "equipe" "técnico de enfermagem" "curativo" "trabalhar" "grande" "cirurgia" "complexidade" "dimensionamento" "então" "hoje" "correto" "realizar" "adequado" "complexidade" "precisar". Ficou evidente na fala do enfermeiro que o trabalho na clinica de ortopedia e traumatologia é realizado em equipe, porém, é notório nas falas a não realização do dimensionamento de pessoal.

(...) "a nossa equipe é muito parceira então nós não temos uma divisão de você fica com esse ou aquele. É técnicos de enfermagem ajudando o outro, a equipe trabalha em parceria nunca individual, todos se ajudam com o curativo, medicações e cuidados" (E3).

(...) “não realizo o dimensionamento de pessoal não aquele que vi na faculdade” (E4).

Apesar de o trabalho em equipe ser extremamente importante, não exclui a necessidade da realização do dimensionamento de pessoal, pois o trabalho em equipe realizado de maneira aleatória, sem o embasamento teórico existe a possibilidade da sobrecarga de trabalho tanto individualmente quanto para a equipe, além disso, favorece a possibilidade de danos adversos ao paciente.

Salta aos olhos a importância do planejamento das ações de enfermagem, sobretudo no gerenciamento, é necessário que sejam utilizados instrumentos que estejam validados de maneira estratégica, que forneçam dados e informações apropriadas para a tomada de decisão e o cálculo da carga de trabalho (Goulart et al., 2014).

Como observado no discurso supracitado o profissional afirma que a faculdade o preparou para realizar o gerenciamento e dimensionamento da UI, porém, o contingente de pessoal disponível na clínica os impede de realizar o dimensionamento de pessoal adequado. Infelizmente o enfermeiro após receber o grau e iniciar sua carreira profissional, acaba conflitando com o que aprendeu e viveu na faculdade e o que vai poder executar na prática, pois não possui recursos humanos suficiente para executar da maneira preconizada pelos manuais de enfermagem a assistência junto ao paciente.

Destacam-se as falas a seguir:

(...) "pois hoje não existe um dimensionamento adequado, o quantitativo de técnicos de enfermagem não me permite realizar o dimensionamento correto com a divisão melhor do trabalho. Eu trabalho com poucos técnicos de enfermagem" (E2).

(...) "não vou negar são apenas dois técnicos de enfermagem na clinica de ortopedia e o paciente de ortopedia é muito dependente na maioria das vezes" (E4).

A casuística citada pelo profissional enfermeiro para a não realização do dimensionamento de pessoal tem sido a realidade de várias unidades de internação nos mais variados hospitais do Brasil (Coelho, 2013).

Observa-se em estudos onde se utilizou o SCP a fim de realizar o dimensionamento adequado de pessoal, que na maioria das UI o número de profissional estava inadequado para a demanda de serviço, o que gerava uma sobrecarga de trabalho na equipe, gerando ainda a falta de motivação profissional, e o consequente decaimento da qualidade da assistência oferecida. 
Como bem expressado na fala do profissional, o paciente ortopédico depende de um cuidado mais elevado da equipe de enfermagem, ou seja, o número de horas de cuidado dispensada a esses pacientes é maior, justamente pelo seu grau de complexidade, por esse paciente fazer uso de fixadores externos, tração esquelética, pinos e imobilização, apresentando limitação que o impede de desenvolver suas atividades diárias de auto cuidado, sendo esse, passado a ser realizado pela equipe de enfermagem.

Ressalta-se que o tempo utilizado na assistência de enfermagem, depende de algumas variáveis como a idade do paciente, sua complexidade e colaboração durante o procedimento do material e recurso humano disponível. Assim o número inadequado de pessoal de enfermagem, interfere no prognóstico do paciente, pois o dimensionamento de pessoal está intimamente relacionado à qualidade da assistência (Moraes; Linch; Souza, 2012).

$\mathrm{Na} \mathrm{COT}$ os cuidados diários com o paciente como: banho e higiene pessoal acabam sendo realizados pelos familiares ou por acadêmicos durante a realização de seu estágio curricular, o que pode ser observado na fala:

(...) "quando tem aluno nós aproveitamos, e eles realizam os curativos menores e os técnicos de enfermagem ficam com os maiores e isso melhora bastante" (E1)

O profissional transparece em sua fala que a presença do acadêmico no ambiente hospitalar causa uma melhora, o alívio na sobrecarga de trabalho, pois é possível dividi-lo com mais pessoas.

A relevância do estagiário na unidade de internação foi enfatizada em outros estudos, o acadêmico torna-se por um período parte da equipe, porém não é o responsável imediato por ela. Há nesse processo o estabelecimento da troca de conhecimento e cria-se uma relação de interdependência, essa favorece a assistência de enfermagem (Negreiros; Lima, 2018).

\section{Classe 5: $O$ reflexo do número reduzido de profissional na assistência}

O software ao fazer análises estatísticas agrupou nesta classe as palavras que foram mais similares "assistência" "falha" "precisar" "complicação" "correto" "técnico de enfermagem" "olhar" "holístico" "sinal". Percebeu-se, no relato do enfermeiro que o técnico de enfermagem exerce papel fundamental na assistência de enfermagem prestada ao paciente, porém, essa pode está apresentando falha em algum momento devido ao elevado número de paciente internado na clínica e ao déficit de pessoal de enfermagem. O que é evidenciado nas assertivas abaixo:

(...) "a sobrecarga de trabalho é grande e isso faz com que haja uma falha na assistência e o técnico de enfermagem não realize o cuidado e a assistência correta ao paciente" (E2).

(...) "as complicações do trauma podem sim estar relacionadas à falha na assistência, e a falha na assistência pode causar danos ao paciente, pois se o curativo ou o procedimento não for realizado de maneira correta o paciente poderá ficar mais tempo internado e pode precisar realizar outro procedimento cirúrgico" (E1).

Neste contexto a sobrecarga de trabalhado mais uma vez é citada pelo profissional enfermeiro, porém, aqui é dado ênfase ao reflexo do número reduzido de profissional na assistência de enfermagem. O enfermeiro é categórico ao afirmar que por falha na assistência o paciente tem danos maiores como à necessidade de uma nova abordagem cirúrgica.

O paciente ortopédico necessita de cuidados mais efetivos, principalmente no pós-operatório, onde a dor apresenta-se de magnitude intensa a moderada, devendo a equipe de enfermagem atentar-se para a analgesia, não apenas medicamentosa, mas através de outros métodos disponíveis para que a enfermagem promova o alívio da dor. Neste momento é de grande importância que o enfermeiro tenha traçado o plano de intervenção. Ressalta-se que a assistência de enfermagem não é responsabilidade apenas do técnico e sim da equipe de enfermagem. 
Além da dor a equipe precisa está capacitada e ter um olhar holístico para identificar outros possíveis problemas apresentados pelo paciente ortopédico como o déficit motor, o sangramento intenso, e a imobilização precoce devido ao risco elevado de tromboembolismo (Veiga et al., 2009).

\section{Classe 6: Orientações direcionadas ao paciente na alta hospitalar}

As palavras que emergiram nessa classe foram "retorno" "orientar" "alta" "medicação" "sinal" "curativo". Antes de o paciente deixar o hospital, o enfermeiro realiza orientações básicas que esclarece sobre os cuidados, as atividades permitidas e as limitações impostas pelo tratamento. Destacam-se as falas a seguir:

(...) "na alta oriento em relação ao retorno ao ambulatório, ao uso da medicação, o cuidado com o curativo, sinais de alerta e o cuidado com o fixador externo se o paciente tiver fazendo uso" (E4).

(...) "gosto muito de enfatizar sobre o retorno ambulatorial pelo fato do paciente morar longe e ter suas dificuldades eu sempre oriento que fique por perto, principalmente os que moram em outros municipios" (E3).

A alta é o momento no qual o paciente deixa o ambiente hospitalar, porém, o mesmo irá dar continuação ao cuidado em seu domicilio. Apesar de o enfermeiro da COT realizar orientações ao paciente, não tem traçado um plano de alta que atenda a necessidade, os medos e anseios do mesmo e de seu familiar, agora cuidador.

Segundo Martins, Silva e Ferraz (2013), no momento da alta o paciente é envolto por vários sentimentos, a volta para o domicílio causa satisfação, porém, também gera medo, pois o mesmo sente-se inseguro por não saber como se dará o cuidado longe da equipe multidisciplinar, uma vez que não há o preparo do familiar/cuidador. Esse medo e insegurança crescem de acordo com o grau de dependência do paciente.

É realidade o fato de o paciente retornar ao seu domicílio cheio de dúvidas e questionamentos que deveriam ter sido sanados no ambiente hospitalar durante o período em que esteve internado, o que observou-se nesse estudo foi que o enfermeiro realiza orientações verbais apenas no momento da alta, não sendo reproduzidas em documento impresso para posterior consulta do paciente e/ou familiar. Corroborando com este estudo Araújo (2012), reforça que no momento da alta é importante sanar todas as dúvidas do paciente, citando o problema de as informações serem ofertadas apenas oralmente.

\section{Conclusão}

Uma assistência qualificada e humanizada depende de diversos fatores, os quais interferem na adoção de formas alternativas de se organizar a assistência de enfermagem, especialmente no que concerne à implantação da SAE, segundo os modelos atualmente adotados pelas instituições hospitalares.

O enfermeiro exerce papel fundamental para que a assistência de enfermagem da unidade hospitalar seja de qualidade e satisfatória para o paciente, fazendo com que o tempo de permanência hospitalar não seja aumentado em consequência de eventos relacionados à negligência do cuidado.

O dimensionamento de pessoal gera implicações diretas no desempenho das competências do enfermeiro e, consequentemente, provoca prejuízo na qualidade da assistência quando em desacordo com o ideal para atender a demanda da clientela assistida. Sua adequação pode ser considerada como fator motivador, importante, para o bom desempenho profissional e para a qualificação da assistência, ressalto que este trabalho visa servi de fontes para novas publicações quanto a temática. 


\section{Referências}

Araújo, F. S. R. (2012). O planejamento da alta hospitalar pelo enfermeiro aos clientes das unidades clínicas e cirúrgicas: perspectiva da complexidade em saúde numa atitude transdisciplinar. Dissertação (Mestrado em Ciências do cuidado em saúde) - Escola de enfermagem Aurora de Afonso Costa, Universidade Federal Fluminense. Niterói. https://app.uff.br/riuff/handle/1/1042.

Bardin, L. (2011). Análise de conteúdo. Edições 70.

Barros, A. L. B. L. \& Lopes, J. L. (2010). A legislação e a sistematização da assistência de enfermagem. Revista Enfermagem em Foco. 1(2), 63-5. http://revista.cofen.gov.br/index.php/enfermagem/article/view/17/18.

Brito, A. P; Guirardello, E. B. (2012) Nível de complexidade assistencial dos pacientes em uma unidade de internação. Revista brasileira de enfermagem. v. 65, n. 1, p. 92-6, http://www.scielo.br/scielo.php?script=sci_arttext\&pid=S0034-71672012000100013.

Coelho, M. A. (2013) Dimensionamento de profissional de enfermagem nas unidades de internação de adultos de um hospital de ensino da região Centro-oeste do Brasil. Tese (Doutorado) - Faculdade de enfermagem, Universidade de Goiás. Goiânia, https://ppgenf.fen.ufg.br/up/127/o/Maria_Alice_Coelho.pdf?1391019483.

COFEN. (2009) Resolução COFEN no 358/2009, de 15 de outubro de 2009. Dispõe sobre a Sistematização da Assistência de Enfermagem e a implementação do Processo de Enfermagem em ambientes, públicos ou privados, em que ocorre o cuidado profissional de Enfermagem, e dá outras providências. In: Conselho Federal de Enfermagem [legislação na internet]. Brasília; http: http://www.cofen.gov.br/resoluo-cofen 3582009_4384.html.

COFEN. (1986) Lei n 7.498/86, de 25 de junho de 1986. Dispõe sobre a regulamentação do exercício da Enfermagem e dá outras providências. In: Conselho Federal de Enfermagem [legislação na internet]. Brasília; http://www.cofen.gov.br/lei-n-749886-de-25-de-junho de-1986_4161.html.

COFEN. (2017) Resolução no 543 de 18 de abril 2017. Atualiza e estabelece parâmetros para o Dimensionamento do Quadro de Profissionais de Enfermagem nos serviços/locais em que são realizadas atividades de enfermagem. In. Conselho Federal de Enfermagem [legislação na internet]. Brasília. http://www.cofen.gov.br/resolucao-cofen 5432017_51440.html.

Ferreira, P. C. et al. (2017). Classificação de paciente e carga de trabalho da enfermagem em terapia intensiva: comparação entre instrumentos. Revista Gaucha de Enfermagem,v. 38, n. 2, p. 1-7, http://www.scielo.br/pdf/rgenf/v38n2/0102-6933-rgenf-1983-144720170262782.pdf

Fugulin, F. M. T. et al. (2012). Tempo de assistência de Enfermagem em Unidade de Terapia Intensiva: avaliação dos parâmetros propostos pela Resolução COFEN n²93/04. Revista Latino-Americana de Enfermagem. v. 20, n. 2, p. 325-32. http://www.scielo.br/scielo.php?pid=S0104$11692012000200015 \&$ script=sci_arttext\&tlng=pt.

Gaidzinski R. R. (1994). O dimensionamento do pessoal de enfermagem segundo a percepção de enfermeiras que vivenciam esta prática. Tese (Doutorado) - Escola de Enfermagem, Universidade de São Paulo. São Paulo. https://repositorio.usp.br/item/000741601.

Goulart, L. L. et al. Carga de trabalho de enfermagem em uma unidade de terapia intensiva de trauma. Revista eletrônica enfermagem, v. 16, n. 2, p. 346- 51, 2014. Disponível em: https://deploy.extras.ufg.br/projetos/fen_revista/v16/n2/pdf/v16n2a10.pdf. Acesso em: 02 Dez. 2019.

Horta, Wanda de Aguiar (1979). O processo de Enfermagem. São Paulo: EPU/Edusp.

Kfuri J. M. (2011). O trauma ortopédico no Brasil. Revista Brasileira de Ortopedia [online], v. 46, suppl 1 [Acessado 15 Novembro 2021] , Disponível em: <https://doi.org/10.1590/S0102-36162011000700003>. Epub 10 Ago. ISSN 1982-4378. https://doi.org/10.1590/S010236162011000700003

Moraes, M; Linch, G.F.C; Souza, E.N. (2012) Classificação de pacientes internados em uma unidade traumatológica. Revista Gaúcha de Enfermagem, v. 33, n. 2, p. 52-59. http://www.scielo.br/pdf/rgenf/v33n2/09.pdf.

Martins, A. C. S; silva, J.G; Ferraz, L.M. (2013). Orientações de enfermagem na alta hospitalar: contribuições para o paciente e cuidadores. . In: Convibra - Congresso Virtual Brasileiro de Administração. anais do Convibra. http://www.convibra.com.br/UPLOAD/PAPER/2013/70/2013_70_7857.PDF.

Negreiros, R. V; Lima, V.C.B. (2018). Importância do estágio supervisionado para o acadêmico de enfermagem no hospital: compartilhando experiências vivenciadas com a equipe de trabalho. Revista Unincor, v. 16, n. $2, \quad$ p. 1- 7. http://periodicos.unincor.br/index.php/revistaunincor/article/view/4359/pdf_819.

Prodanov, C. C.; Freitas, E. C. (2013). Metodologia do trabalho científico [recurso eletrônico]: métodos e técnicas da pesquisa e do trabalho acadêmico. Novo Hamburgo: Feevale.

Tannure, M.C; Pinheiro, A.M. (2013). Sistematização da Assistência de Enfermagem. 2. ed. Rio de Janeiro: Guanabara Koogan, 2013.

Veiga V. Rojas SSOR, Morais EASM, Santos ECA, Cruz OO, Marchesini AM, et al. (2009) Protocolo pós- operatório de cirurgia ortopédica. 2009. http://www.ineti.med.br/pdf\%5Cdiretrizes\%5Cdiretrizes20.pdf

Vigna, C. P; Perroca, M.G. (2019). Utilização de sistema de classificação de pacientes e métodos de dimensionamento de pessoal de enfermagem. Revista Arq Ciênc Saúde. v. 14, n.1, p. 8-12, 2007. http://repositorio-racs.famerp.br/racs_ol/vol-14-1/id215.pdf. 